
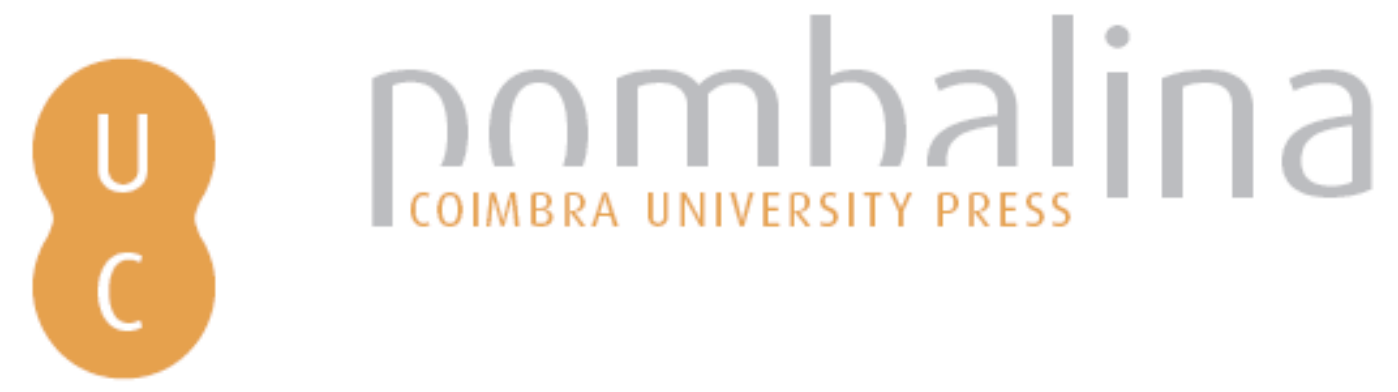

\title{
Mercado laboral y formación profesional: programas de educación continua y necesidad de formación especializada en Ciencias de la Información y Documentación
}

\author{
Autor(es): $\quad$ Atenas Rivera, Javiera \\ Publicado por: Imprensa da Universidade de Coimbra \\ URL \\ persistente: \\ URI:http://hdl.handle.net/10316.2/31927 \\ DOI: \\ DOl:http://dx.doi.org/10.14195/978-989-26-0319-3_31
}

Accessed : $\quad$ 26-Apr-2023 10:24:23

A navegação consulta e descarregamento dos títulos inseridos nas Bibliotecas Digitais UC Digitalis, UC Pombalina e UC Impactum, pressupõem a aceitação plena e sem reservas dos Termos e Condições de Uso destas Bibliotecas Digitais, disponíveis em https://digitalis.uc.pt/pt-pt/termos.

Conforme exposto nos referidos Termos e Condições de Uso, o descarregamento de títulos de acesso restrito requer uma licença válida de autorização devendo o utilizador aceder ao(s) documento(s) a partir de um endereço de IP da instituição detentora da supramencionada licença.

Ao utilizador é apenas permitido o descarregamento para uso pessoal, pelo que o emprego do(s) título(s) descarregado(s) para outro fim, designadamente comercial, carece de autorização do respetivo autor ou editor da obra.

Na medida em que todas as obras da UC Digitalis se encontram protegidas pelo Código do Direito de Autor e Direitos Conexos e demais legislação aplicável, toda a cópia, parcial ou total, deste documento, nos casos em que é legalmente admitida, deverá conter ou fazer-se acompanhar por este aviso.

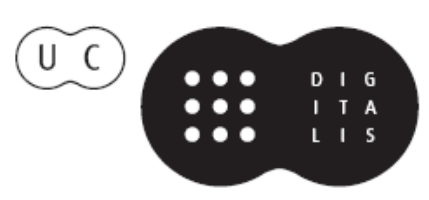


Maria Manuel Borges

Elias Sanz Casado

Coordenação

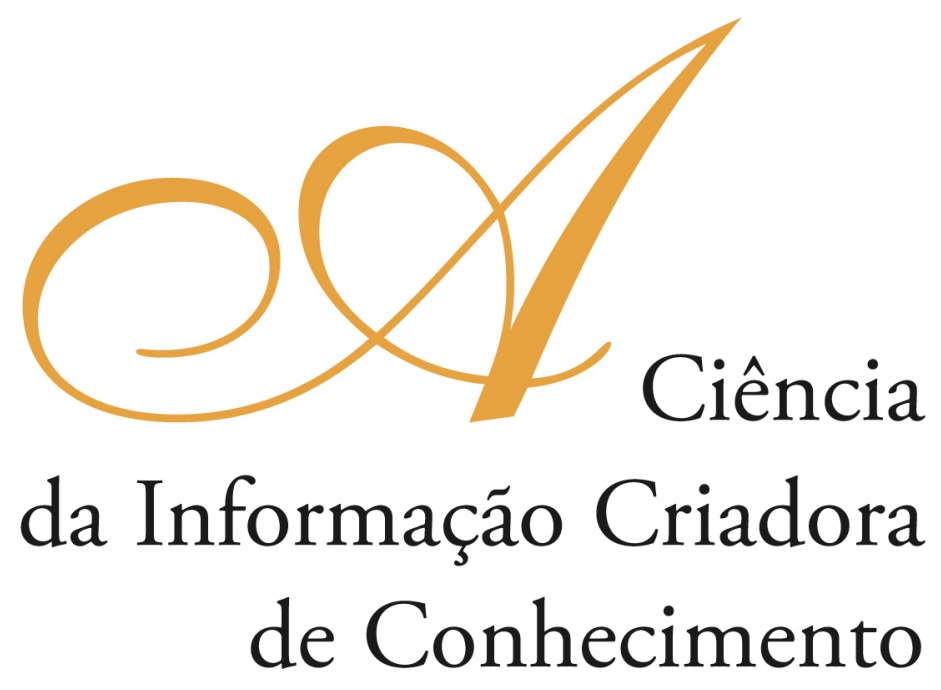

Vol. I

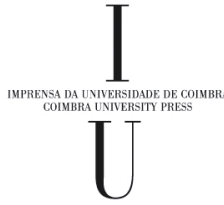

- COIMBRA 2009 


\title{
Mercado laboral y formación profesional: Programas \\ DE EDUCACIÓN CONTINUA Y NECESIDAD DE FORMACIÓN \\ ESPECIALIZAda EN CienCias de la INFORMaCión \\ y Documentación
}

Javiera Atenas Rivera

Universidad de Barcelona (Espanha)

City and Islington College (Reino Unido)

\begin{abstract}
The information society requires permanent changes in the training methods in academic education, in the library and information science case, these changes are related to the labour market requirements and in European case with the Bologna agreement. The market has increased the need to recruit specialized professionals in different knowledge areas, we can observe it trough the job offers that reflect the organizations need seeking specialized professional profiles. The labour market is looking for professional profiles that are able to adapting to new technologies and this is currently a cross professional challenges, to keep pace with the knowledge and, for information professionals one of the best options for continuing education is distance learning. As mentioned Hadengue (2004) «E-learning Strategies seems to offer more information than traditional training because is easier access to information»
\end{abstract}

\section{Objetivos y Metodología}

Los objetivos de este estudio son:

- Determinar el grado de necesidad de implementar postgrados en áreas específicas del conocimiento, ya que la sociedad exige profesionales capaces de entregar un mejor soporte y respuesta a las necesidades de información.

- Encontrar un modelo de formación acorde con las necesidades económicas y sociales de España, Portugal e Iberoamérica, analizando distintos modelos de educación continua para diseñar una propuesta sustentable desde el punto de vista de combinar trabajo y estudios.

Dentro del ámbito Europeo, la formación profesional de los bibliotecarios y documentalistas se encamina más y más hacia ciertas áreas como gestión, administración y tecnologías de la información y la comunicación, sin embargo, el mercado como consecuencia del modelo económico imperante, comienza a exigir cada vez más profesionales, que, en conjunto con áreas anteriormente mencionadas, sean expertos en ciertos ámbitos como medicina, artes, derecho, ciencias puras y ciencias aplicadas.

Un ejemplo de la oferta de formación profesional especializada en el contexto legal, es el de la Universidad de Denver, EEUU, que a partir de septiembre de 2009 implantara un cursos para bibliotecarios otorgando el Grado de MLIS con especialización 
en Derecho, lo que destaca en este programa, es que esta diseñado para profesionales que trabajan y que quieren especializarse en su carrera.

Este trabajo contempla fases de análisis cuantitativo para entender las necesidades del mercado laboral en Europa y Estados Unidos, para lo que han sido revisadas:

- 120 ofertas de trabajo de Alemania, Gran Bretańa y Estados Unidos.

- Periodo: enero - marzo 2009

- 20 planes de estudios de postgrado de escuelas de ciencias de la información de Alemania, España, Francia, Gran Bretaña y Estados Unidos.

Los parámetros de análisis de las ofertas de trabajo son:

- País de la oferta

- Tipo de organización (publica - privada)

- Grado de especialización solicitado (general - específico)

- Área de la biblioteca

- Área del conocimiento

- Competencias técnicas

Los parámetros para analizar los planes de estudio son los siguientes:

- País de la institución

- Modalidad (presencial - a distancia)

- Área del conocimiento

- Grado de especialización (Diploma - Postgrado - Master - Doctorado)

Para obtener ofertas de trabajo de distintos países se utilizaron los siguientes sitios Web:

UK - INFOmatch CILIP

US: ALA Joblist - LisJobs

Germany : Stellenservice Bibliothekswesen

Esta parte de la investigación se realiza utilizando analizando ofertas de trabajo. La técnica usada en la primera parte de este capítulo consiste en la revisión y análisis descriptivo, de 120 ofertas de trabajo aparecidas en sitios del Web de las asociaciones de bibliotecarios $\mathrm{u}$ otras relacionadas con esta disciplina. Las ofertas fueron seleccionadas en cinco países, Alemania, Reino Unido, Estados Unidos Los países fueron elegidos porque cada uno de ellos tiene una realidad diversa que se manifiesta tanto en la forma en que contratan personal o cómo distribuyen el trabajo que ofrecen. El análisis se ha hecho utilizando un software de encuestas, lo que permite para ir completando los requerimientos de 6 parámetros para obtener datos concretos que permitan obtener un perfil profesional y así poder revisar las necesidades de formación profesional que tienen los profesionales en las distintas áreas de las ciencias de la información.

En el caso del análisis geográfico las realidades más similares son el Reino Unido y los Estados Unidos, porque, la lista de distribución principal de las ofertas del trabajo pertenece a sus asociaciones nacionales de biblioteca.

En el caso de EE.UU. está el sitio Web de la ALA (ALA) que distribuye la mayoría de las ofertas del trabajo para este país y ofrece también las oportunidades de trabajo para Canadá, el Oriente Medio e Israel, lo que significa que se enfoca, no solamente 
en el alcance local, sino que, proporciona las oportunidades de trabajo para la gente de otros lugares y promueve la movilidad entre diversos países se, estas se seleccionaron aquellas que tenían claramente definido el perfil profesional que buscaban. Para este estudio, se tomaron solamente las ofertas para EE.UU., debido a lo difícil de encontrar capacidades básicas y homogéneas cuando las ofertas del trabajo fueron fijadas para otros países, y sobre todo en requerimientos extremadamente complejos como "Bibliotecario de madre lengua hebrea", por lo que no se han estudiado este tipo de ofertas con requisitos demasiado específicos ya que no pueden ser comparadas con otras.

En el caso del Reino Unido, las ofertas, fueron seleccionadas del sitio Web del Chartered Institute for Library and Information Professionals (Cilip) que ofrece puestos de trabajo para Inglaterra, Escocia, País de Gales e Irlanda del Norte. Las ofertas del trabajo comprenden una amplia gama de posiciones con diversos niveles de cargos como auxiliares, bibliotecarios asistentes, gestores de información y jefe de bibliotecas entre otras.

Las ofertas de trabajo de Alemania fueron tomadas del sitio Web de Stellenservice Bibliothekswesen, uniste sitio Web especializado en trabajo que ofrece puestos para Alemania, Austria y Suiza. Fue seleccionado porque las ofertas se presentan claramente y contiene bastante información para obtener datos exactos de ellas.

Cada oferta fue analizada de trabajo fue analizada para obtener información que nos permite entender que quiere el mercado de trabajo, cual es el perfil que busca, en el caso de las áreas de la biblioteca y si estos requerimientos están siendo cubiertos por los programas de educación continua de las universidades en los respectivos espacios geográficos, por lo que se han analizado 20 programas de educación de post grado en ciencias de la información, ya sea presenciales, a distancia o mixtos en áreas relacionadas con la documentación y en áreas especificas del conocimiento, enfocándonos en los resultados obtenidos en el análisis de las ofertas de trabajo.

En el caso de la revisión de programas de especialización de postgrado, el análisis se llevo a cabo analizando 20 programas en 17 universidades de un amplio segmento geográfico bajo diversos puntos de vista, considerando si los estudios se impartían en de manera presencial y a distancia, el área del conocimiento que abarcaban y el grado de especialización.

\section{Conclusiones: Análisis del mercado de trabajo y de los programas de educación en Ciencias de la Información}

Cada país refleja su mercado de y para este estudio hemos analizado las 3 realidades que mejor reflejan el contexto económico actual y en las cuales des mas fácil recuperar información, ya que son las mismas asociaciones de bibliotecarios y documentalistas los que gestionan la publicación y difusión de ofertas de trabajo. Para este estudio, el 36\% de las ofertas de trabajo vienen de instituciones de Estados Unidos (debe considerarse que el índice de población es superior al Reino Unido y Alemania). 


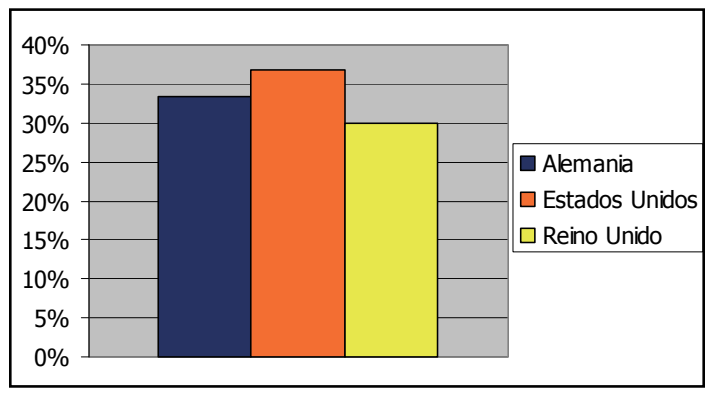

La mayor parte de las bibliotecas que han reflejado sus necesidades de profesionales especializados a través de ofertas de trabajo con las bibliotecas académicas, sobre todo en aquellas áreas universitarias científicas donde el apoyo de los bibliotecarios en resolver dudas especificas en áreas determinadas es imprescindible y por lo tanto, la capacidad de actualización de estos profesionales es una constante. Las bibliotecas especializadas, por motivos obvios son el segundo rango de instituciones que buscan profesionales que ya tengan conocimientos en áreas determinadas. Los organismos privados, sobre todos aquellos ligados a la industria farmacéutica y legal buscan a la vez profesionales altamente capacitación en sus áreas de mercado por que el costo de entrenamiento es demasiado alto para las empresas privadas ya que la capacitación suele ser externalizadas en instituciones de educación superior con un alto costo en ausentismo y con tasas institucionales.
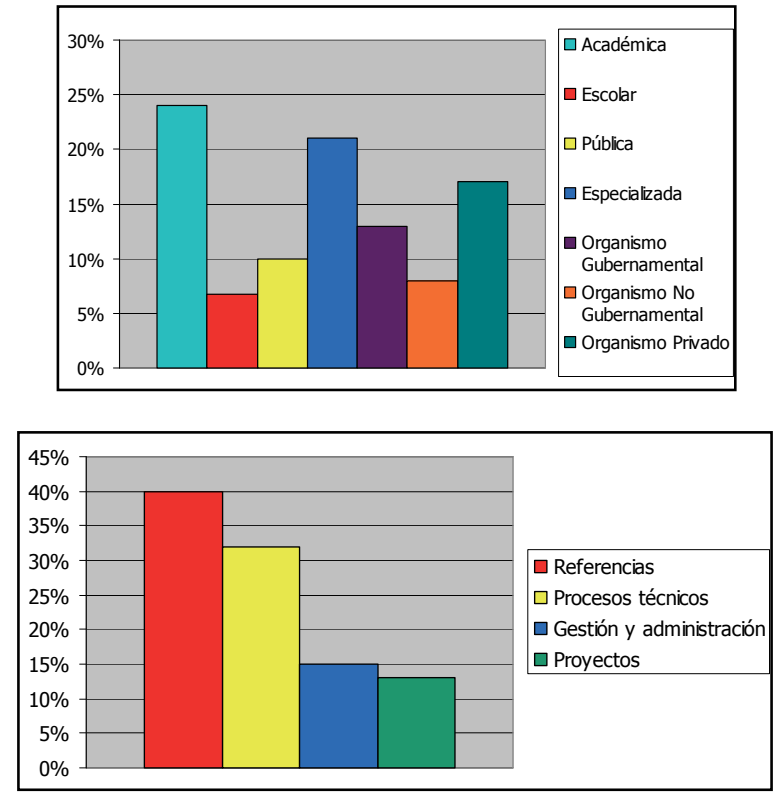

Las áreas de referencias y procesos técnicos son aquellas que exigen profesionales especializados en áreas particulares, por que son los que deben manejar las temáticas con mayor precisión. Un referencista, además de conocer ciertas áreas de manera 
profunda, deben hablar mas un idioma, sobre todos aquellos donde su disciplina se destaca más, y esto se refleja en ofertas con requisitos como "bibliotecario referencista para departamento de hispanidades en universidad de Estados Unidos, requisitos Ingles y Español, conocimientos de bases de datos y conocimientos de literatura hispánica”.

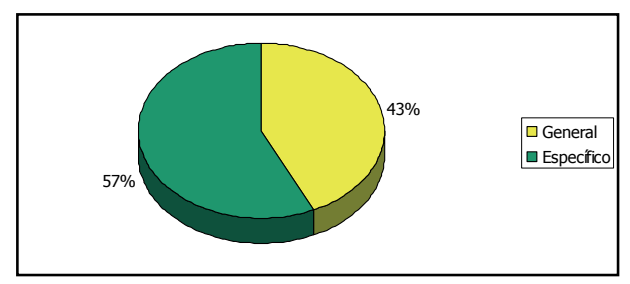

El mercado de trabajo a través de sus herramientas de reclutamiento de personal, refleja sus necesidades y en el caso de los bibliotecarios 57\%, busca profesionales especializados, debemos considerar que en algunos países de Europa como es Estados existe tomar la licenciatura en Ciencias de la Información luego de haber hecho una diplomatura en otra área del conocimiento y viceversa, lo que facilita a las organización poner requisitos mas específicos en sus ofertas de trabajo, sin embargo, la mayoría de los bibliotecarios y documentalista son profesionales que no tienen otra formación que la de Ciencias de la Documentación y que esta sociedad de la información refleja mas y mas la necesidad de profesionales especializados, no solo en el área de las bibliotecas, sino que en áreas tan diversas como la economía, las tecnologías e ingenierías, por lo que la formación profesional debe considerar este punto al planear los programas de estudio, sobretodo en los programas de formación continua.

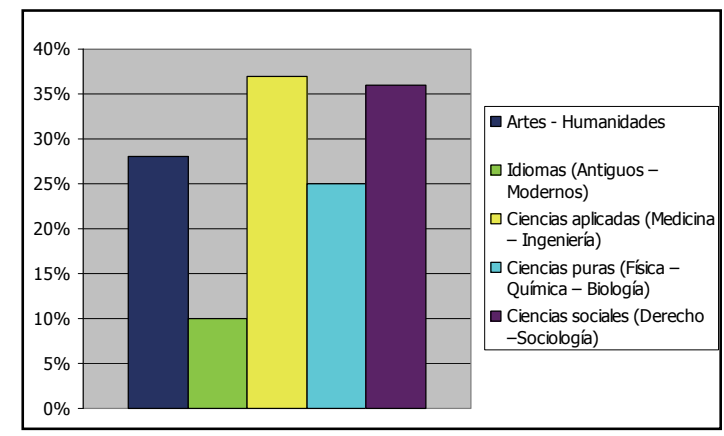

Como podemos ver en los gráficos que reflejan las ofertas de trabajo, cada vez mas instituciones sobre todo, las bibliotecas Universitarias y Especializadas buscan un perfil profesional con conocimientos profundos en áreas especificas, sobre todo, cuando las instituciones buscan un referencista en Artes, Humanidades, Ciencias Aplicadas (medicina - ingeniería). La necesidad de formar profesionales especializados en áreas específicas del conocimiento, queda en manifiesto con el análisis de las ofertas del trabajo, en las cuales se refleja que mas de un $60 \%$ de las organizaciones que buscaban profesionales en áreas como medicina, ciencias o derecho, buscan un perfil que sea altamente especializado, sobretodo en Derecho y Medicina que son las áreas mas solicitadas. 
En cuanto a las competencias técnicas que busca el mercado laborar, de debe considerar que como muchas de las ofertas que buscaban un profesional especializado en ciertas áreas precisas del conocimiento, son para puestos de referencia, las capacidades mas requeridas son aquellas relacionadas con el manejo de bases de datos.

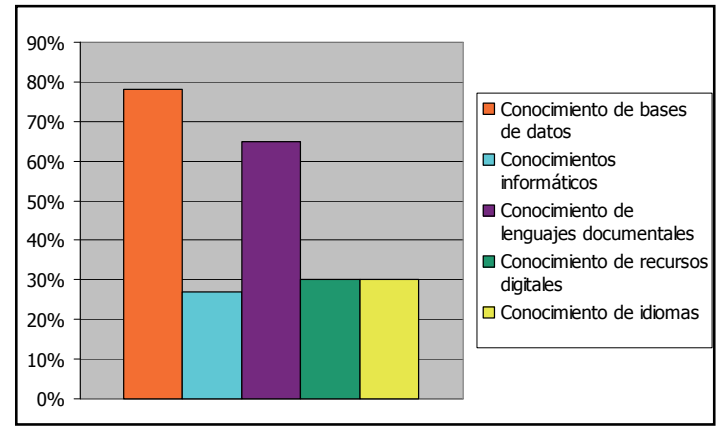

Si bien las organización y empresas buscan profesionales altamente calificados en áreas especificas del conocimiento, solo un $20 \%$ de los programas de masters es de carácter específico como leyes o algún campo de las ciencias por lo tanto se observa una carencia en el mercado de profesionales especializados indica una necesidad urgente, sobretodo en Europa de desarrollar programas de educación continua en áreas especificas del conocimiento para suplir la demanda de personal calificado.

En cuanto a la formación de post grado en Ciencias de la información el modelo presencial sigue siendo predominante, sin embargo España esta un paso adelante que otros países Europeos como el Reino Unido. Estados Unidos presenta un número importante, casi del $50 \%$ de programas de formación continua en módulos a distancia. Dentro de España el sistema de educación a distancia para profesionales que quieren especializarse en un área concreta, un modelo es Instituto de Formación Continua de la Universitat de Barcelona (IL3) y como este instituto ha ido desarrollando modelos de formación a distancia en áreas tan disímiles como la museografía interactiva y la medicina.

Dentro de los modelos de educación a distancia, el Reino Unido destaca por el numero de empresas privadas dedicadas a desarrollar módulos online en una amplia gama de temas generales desde atención al cliente a leyes, muchos de ellos en formatos de SCORM que son utilizados por diferentes instituciones para mejorar las competencias de sus profesionales, incrementar la productividad sin tener que salir de las dependencias corporativas y ahorrando en consultores externos para capacitar personal.

El E-learning se puede considerar como un modelo sustentable con las economías modernas a la hora de capacitar personal ya que el índice de perdida económica por ausentismo laboral se ve disminuido al ceder a los profesionales un rango de horas a la semana para dedicarse a los estudios estando presente en las dependencias de las organizaciones. Como el sistema de educación remota requiere constancia, es esencial el apoyo de los equipos de trabajo, sin embargo, al ser los cursos dinámicos y constantes, los conocimientos se pueden ir poniendo poco a poco en práctica durante el periodo de estudios. Las empresas y las personas se benefician de los procesos de capacitación a través de modelos de educación a distancia, pudiendo los bibliotecarios 
convertirse en especialistas sobre un área del conocimiento sin tener que sacrificar el puesto de trabajo ni los ingresos y las organizaciones desde el punto de vista que no tienen que buscar en reemplazante mientras dura el periodo de estudios y que los cambios pueden ser a corto plazo dependiendo de la duración de los cursos, pero como formato sustentable, actualmente es el modelo que mejor se adapta a las necesidades económicas actuales donde los profesionales deben estar altamente calificados en áreas especificas del conocimiento y las empresas buscan personal dinámicos que puedan ir actualizando sus conocimientos de manera practica efectiva y donde el sistema educacional tiene un mercado que cubrir para ser un ente catalizador entre las necesidades económicas de la sociedad del conocimiento y las necesidades e desarrollo profesional de las personas.

\section{Referências bibliográficas}

Akeroyd, J. (2005). Information management and e-learning: Some perspectives. Aslib Proceedings, 57(2), 157-167.

Hadengue, V. (2004). What can e-learning do for university libraries? Library Review, 53(8), 396-400.

Homan, G., \& Macpherson, A. (2005). E-learning in the corporate university. Journal of European Industrial Training, 29(1), 75-90.

Sharifabadi, S. (2006). Ow digital libraries can support e-learning. The Electronic Library, 24(3), 389-401.

Wiston, M., \& Dunkley, L. (2002). Leadership Competencies for Academic Librarians: The Importance of Development and Fund-raising. College \& Research Libraries, 172, 200-216. Retrieved July 15, 2009, from http://www.ala.org/ala/acrl/acrlpubs/crljournal/backissues2002b/ march02/winston.pdf

Wilson, T. (2000, February 15). World list of departments and schools of information studies, information management, information services, etc. Retrieved July 15, 2009, from http:// informationr.net/wl/index.html

Wittwer, R. (2000). Special Libraries - How to Survive in the Twenty-First Century,. The Electronic Library, 19(4), 221-224. 